\title{
Vasospasm on transcranial Doppler is predictive of delayed cerebral ischemia in aneurysmal subarachnoid hemorrhage: a systematic review and meta-analysis
}

\author{
Gyanendra Kumar, MD, ${ }^{1}$ Reza Bavarsad Shahripour, MD, ${ }^{1}$ and Mark R. Harrigan, MD1,2 \\ ${ }^{1}$ Comprehensive Stroke Center, Department of Neurology, and 2Department of Neurosurgery, University of Alabama at \\ Birmingham, Alabama
}

OBJECTIVE The impact of transcranial Doppler (TCD) ultrasonography evidence of vasospasm on patient-centered clinical outcomes following aneurysmal subarachnoid hemorrhage (aSAH) is unknown. Vasospasm is known to lead to delayed cerebral ischemia $(\mathrm{DCl})$ and poor outcomes. This systematic review and meta-analysis evaluates the predictive value of vasospasm on $\mathrm{DCl}$, as diagnosed on TCD.

METHODS MEDLINE, Scopus, the Cochrane trial register, and clinicaltrials.gov were searched through September 2014 using key words and the terms "subarachnoid hemorrhage," "aneurysm," "aneurysmal," "cerebral vasospasm," "vasospasm," "transcranial Doppler," and "TCD." Sensitivities, specificities, and positive and negative predictive values were pooled by a DerSimonian and Laird random-effects model.

RESULTS Seventeen studies ( $n=2870$ patients) met inclusion criteria. The amount of variance attributable to heterogeneity was significant $\left(1^{2}>50 \%\right)$ for all syntheses. No studies reported the impact of TCD evidence of vasospasm on functional outcome or mortality. TCD evidence of vasospasm was found to be highly predictive of DCl. Pooled estimates for TCD diagnosis of vasospasm (for DCI) were sensitivity 90\% (95\% confidence interval [Cl] 77\%-96\%), specificity $71 \%$ (95\% Cl 51\%-84\%), positive predictive value $57 \%$ (95\% Cl 38\%-71\%), and negative predictive value $92 \%(95 \% \mathrm{Cl}$ $83 \%-96 \%)$.

CONCLUSIONS TCD evidence of vasospasm is predictive of $\mathrm{DCl}$ with high accuracy. Although high sensitivity and negative predictive value make TCD an ideal monitoring device, it is not a mandated standard of care in aSAH due to the paucity of evidence on clinically relevant outcomes, despite recommendation by national guidelines. High-quality randomized trials evaluating the impact of TCD monitoring on patient-centered and physician-relevant outcomes are needed.

http://thejns.org/doi/abs/10.3171/2015.4.JNS15428

KEY WORDS aneurysmal subarachnoid hemorrhage; cerebral vasospasm; meta-analysis; delayed cerebral ischemia; transcranial Doppler; vascular disorders; mean flow velocity

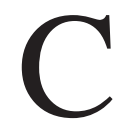
EREBRAL vasospasm is a major cause of poor outcomes in aneurysmal subarachnoid hemorrhage (aSAH), resulting in cerebral ischemia, infarction, disability, and death. ${ }^{20,25}$ Cerebral vasospasm is noted on angiography in as many as $70 \%$ of patients following aSAH and is predicted by high mean flow velocities (MFVs) on transcranial Doppler (TCD) ultrasonography..$^{19,24,26,35}$ Vasospasm is strongly associated with delayed cerebral ischemia (DCI) ${ }^{12}$ and cerebral infarction, ${ }^{11,39,49}$ although poor outcomes can still occur in its absence. Of the current techniques available for diagnosing cerebral vasospasm, catheter angiography and CT angiography are invasive, and require contrast-dye injection and radiation exposure, and therefore do not allow dynamic monitoring of vasospasm. ${ }^{31}$ The radiation exposure and invasive nature of angiography implies that it is not useful for identifying subclinical vasospasm prior to the onset of the condition, and that its use is frequently restricted to confirm prevalent vasospasm

ABBREVIATIONS aSAH = aneurysmal subarachnoid hemorrhage; $\mathrm{Cl}=$ confidence interval; $\mathrm{DCl}=$ delayed cerebral ischemia; $\mathrm{df}=$ degrees of freedom; $\mathrm{MFV}=$ mean flow velocity; $M W E=$ mean weighted estimate; $N P V=$ negative predictive value; $P P V=$ positive predictive value; $T C D=$ transcranial Doppler.

SUBMITTED February 24, 2015. ACCEPTED April 8, 2015.

INCLUDE WHEN CITING Published online October 23, 2015; DOI: 10.3171/2015.4.JNS15428. 
in patients who have already progressed to a symptomatic status. Therefore, despite knowledge that vasospasm can be fatal after aSAH and despite available treatments, opportunities to reduce the incidence of symptomatic vasospasm are lost, and it is often left untreated because it frequently remains undetected by the providers. ${ }^{52} \mathrm{TCD}$, on the other hand, provides a noninvasive, safe, and bedside modality for dynamic assessment and monitoring of vasospasm. Unlike angiography, TCD can predict symptomatic vasospasm and has high sensitivity, specificity, and posi-

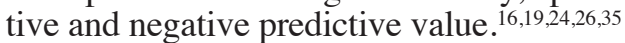

Although approved and recommended by the American Heart Association/American Stroke Association (rated Class IIA/Level B evidence), American Academy of Neurology, and Neurocritical Care Society (moderate quality evidence/strong recommendation) as a safe and effective modality for noninvasive daily monitoring in aSAH, lack of knowledge of the impact on patient-centered outcomes (disability, quality of life, stroke, mortality, etc.) and weak strength of evidence has prevented it from becoming mandated as a standard of care.$^{5,7,43}$ Consequently, this lack of quality evidence has led to inconsistent and unpredictable utilization of this important resource in neurological intensive care units. ${ }^{4,21,28}$ No prior systematic reviews/metaanalyses have addressed the impact of TCD evidence of vasospasm on patient-centered outcomes. This systematic review and meta-analysis sought to evaluate the clinical impact and predictive value of TCD evidence of vasospasm with the hypothesis that TCD evidence of vasospasm impacts clinical outcomes (i.e., DCI, functional outcome, and mortality).

\section{Methods}

A predesigned protocol was used for a search of the literature, study selection, data synthesis, and sensitivity analysis, adhering closely to the Meta-analysis of Observational Studies in Epidemiology (MOOSE) guidelines. ${ }^{45}$

\section{Search Strategy and Study Selection}

MEDLINE, Scopus, the Cochrane Library, and clinicaltrials.gov were searched through September 2014 to identify relevant studies in the literature with the following key words and terms: "subarachnoid hemorrhage," "aneurysm," "aneurysmal," "cerebral vasospasm," "vasospasm," "transcranial Doppler," and "TCD." Titles and abstracts were screened and articles retrieved if they were relevant or if there was uncertainty. Bibliographies of identified studies as well as relevant reviews in the field were manually searched for potentially eligible studies. Full texts were reviewed when a decision about inclusion could not be made by screening the title and abstract. All study designs (retrospective and prospective) were allowed. Observational studies reporting at least 10 consecutive patients were included. Studies reporting on TCD vasospasm where data pertaining to at least 1 relevant outcome (DCI, functional outcome, or mortality) could not be parsed were excluded. Studies that did not report velocities in $\mathrm{cm} / \mathrm{sec}$ were excluded. Studies that reported fewer than 10 patients with aSAH, regardless of the total number of patients in the study, were excluded. Other exclu- sion criteria were as follows: publication of abstracts only, case reports, letters, comments, reviews, or meta-analyses only; animal studies; languages other than English; duplicate studies; no intervention of interest (TCD); lack of outcomes of interest (DCI, functional outcome, or mortality); and not the population of interest (only adults $>18$ years old). Studies were also excluded if data pertaining to sensitivity, specificity, positive predictive value (PPV), and negative predictive value (NPV) were lacking for any outcome of interest.

\section{Data Extraction and Study Quality}

Two authors (G.K. and R.B.S.) independently abstracted data into a standard data form. Data pertaining to sensitivity, specificity, PPV and NPV, DCI, mortality, and functional outcome were extracted. After ensuring accuracy of the extracted data, the 2 data forms were merged into a single form. Study authors were not contacted for clarification and unpublished data or data from abstracts were not sought.

TCD evidence of vasospasm was defined as MFV $\geq$ $120 \mathrm{~cm} / \mathrm{sec}^{1}$ and Lindegaard ratio $\geq 3 .{ }^{23}$ If the study used another definition of vasospasm but where data were available, TCD vasospasm was recalculated as MFV $\geq 120 \mathrm{~cm} /$ sec. When no data were available to recalculate MFV, the definition of vasospasm in the study was followed. Angiographic confirmation was not required. DCI was defined as clinical or radiological (CT/MRI) evidence of ischemia. ${ }^{50}$ Functional outcome was defined as alive and independent at follow-up. Any follow-up from 30 days to 6 months was allowed.

In addition, data pertaining to trial quality and design were extracted and tabulated. All studies were evaluated for quality using a 6 -category scoring system $(0-6) \cdot{ }^{17}$ The categories were: 1) definitions of inclusion and exclusion criteria; 2) reported aSAH severity; 3 ) aSAH severity defined by a standard grading system (for example, the Fisher scale); 4) DCI clearly defined; 5) TCD vasospasm defined; and 6) reported functional outcome/mortality. For each item, studies received a score of 1 if the study fulfilled the criteria and 0 otherwise. Since the focus of the study was to evaluate the impact of TCD evidence of vasospasm on clinical outcomes, data pertaining to angiographic confirmation were not sought.

\section{Statistical Analysis}

\section{Publication Bias}

Publication bias was evaluated with Begg's and Egger's tests (significance defined as $\mathrm{p}<0.05)^{2,10}$ Funnel plots were generated for visual appraisal of publication bias. Trim and fill algorithm analysis was planned a priori for adjusting the effect of publication bias on the pooled estimate ${ }^{9,37}$ in the event that publication bias was significant.

\section{Heterogeneity}

Heterogeneity between the studies was calculated using the chi-square test for heterogeneity (Cochran Q test). A $\mathrm{p}$ value $<0.5$, corresponding to $\mathrm{Q}>$ degrees of freedom (df), was regarded as significant for detection of statistical heterogeneity. Heterogeneity was quantified using $\mathrm{I}^{2}\left(\mathrm{I}^{2}=\right.$ 
[Q-df] $\times 100 / \mathrm{Q}$, where $\mathrm{df}=\mathrm{k}-1, \mathrm{Q}=$ Cochran $\mathrm{Q}, \mathrm{k}=$ number of studies), ${ }^{18}$ and between-study variance expressed as $\tau^{2}$. Significant heterogeneity was regarded as $\mathrm{I}^{2}>50 \%$.

\section{Data Synthesis}

Data were transformed to Fisher's z and standard errors calculated. These were then transformed back during meta-analysis, yielding mean weighted estimates (MWEs) with $95 \%$ confidence intervals (CIs). A DerSimonian and Laird random-effects model was used to synthesize data for all analyses (the random-effects model defaulted to a fixed-effects model when heterogeneity was absent). ${ }^{6}$ Forest plots were generated to illustrate synthesis. Pooled estimates were expressed as MWEs and 95\% CIs.

\section{Sensitivity Analyses}

Sensitivity analyses were planned a priori to account for heterogeneity. Subgroup syntheses were performed based on the definition of DCI (clinical vs radiological). A quality score was used for a priori-designed sensitivity analysis for study bias integration. A quality score was incorporated into the synthesis in Doi and Thalib's quality-effects regression model, yielding quality-adjusted pooled estimates. ${ }^{8}$ To test the robustness of the syntheses, exclusion sensitivity analysis was performed to evaluate the extent to which each trial influenced the result of the synthesis. For this analysis each study was excluded, one at a time, and the combined value without a particular study calculated. All analyses were performed on Mix 2.0 Pro statistical package (BiostatXL).

\section{Results}

The search strategy and study selection are outlined in the flow diagram (Fig. 1). Seventeen studies ( $n=2870$ patients) met inclusion criteria. ${ }^{3,13,15,16,22,27,29,30,33,34,39-41,44,46,47,51}$ Study characteristics are summarized in Table 1. No studies addressed the comparative impact of TCD vasospasm on functional outcome and mortality. Only 5 studies used the Lindegaard ratio for defining TCD vasospasm. ${ }^{15,27,34,40,41}$ Therefore, to maintain comparability across studies, MFV alone was used to define vasospasm on TCD. No publication bias was found in any synthesis except for the synthesis of NPV ( $p=0.03$, Begg's test). The amount of variance attributable to heterogeneity was significant $\left(\mathrm{I}^{2}>50 \%\right)$ for all syntheses. TCD vasospasm was found to predict DCI with high sensitivity $(90 \%)$ and NPV (92\%), fair specificity (71\%), and poor PPV (57\%; Table 2). Funnel and forest plots are shown in Figs. 2 and 3, respectively.

\section{Sensitivity Analysis}

Estimates were robust to quality adjustment. Subgroup analysis, based on how DCI was defined (clinically vs radiologically), explained heterogeneity. Trends in $\tau^{2}$ explained and accounted for heterogeneity. Studies that defined DCI radiologically were identified as a source of het-

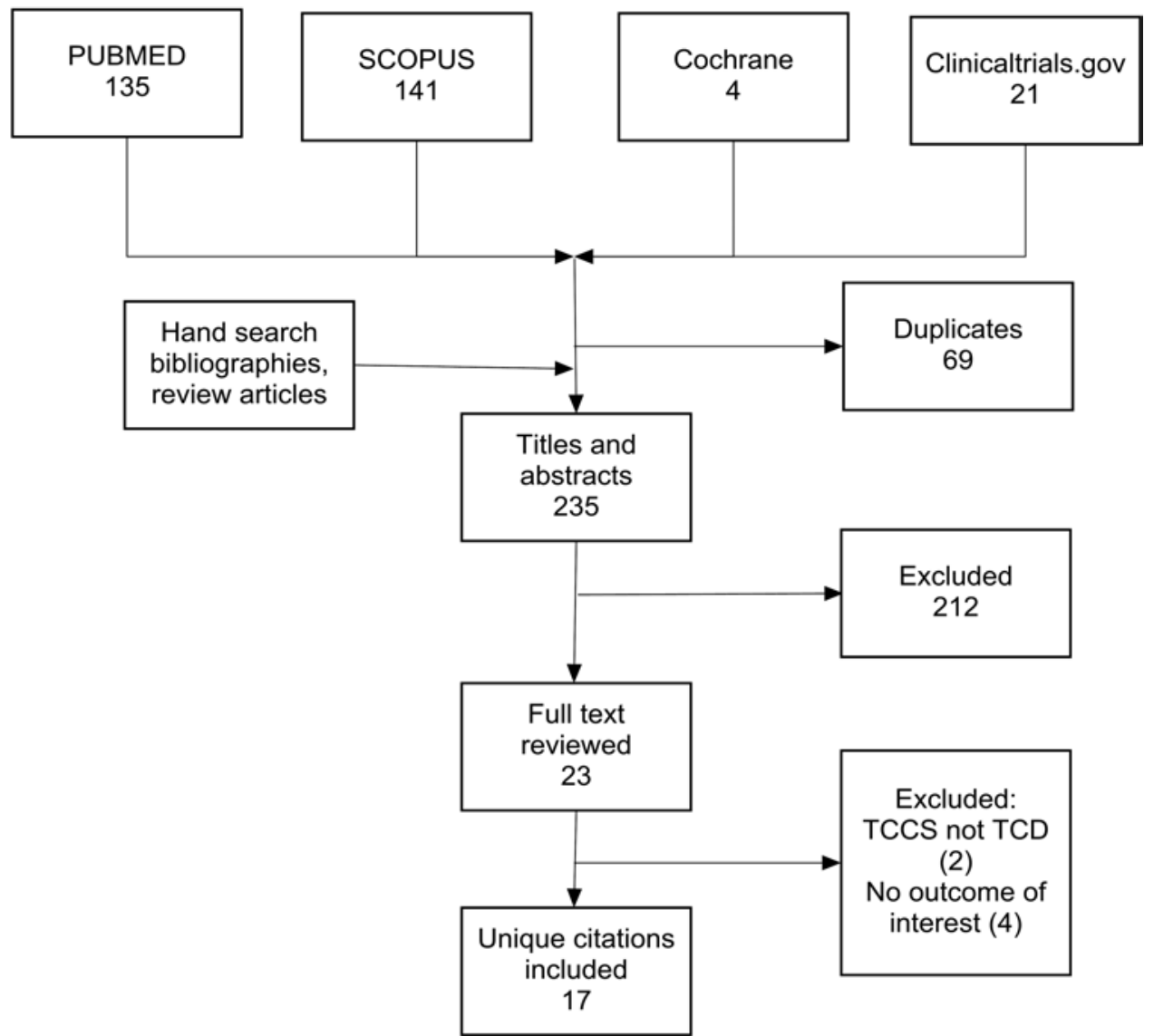

FIG. 1. Flowchart of the literature search and study selection. TCCS = transcranial color-coded sonography. 
TABLE 1. Study characteristics

\begin{tabular}{|c|c|c|c|c|c|c|c|c|c|c|}
\hline Authors \& Year & Design & $\begin{array}{c}\mathrm{DCl} \\
\text { Definition }\end{array}$ & $\begin{array}{c}\text { MFV } \\
\text { Cutoff* }\end{array}$ & $\begin{array}{l}\text { Inclusion/ } \\
\text { Exclusion } \\
\text { Defined }\end{array}$ & $\begin{array}{c}\text { Reported } \\
\text { aSAH } \\
\text { Severity }\end{array}$ & $\begin{array}{c}\text { Standardized } \\
\text { Severity Scale } \\
\text { Used }\end{array}$ & $\begin{array}{c}\text { Defined } \\
\mathrm{DCl}\end{array}$ & $\begin{array}{c}\text { Defined } \\
\text { Vasospasm }\end{array}$ & $\begin{array}{l}\text { Functional } \\
\text { Outcome/ } \\
\text { Mortality }\end{array}$ & $\begin{array}{l}\text { Quality } \\
\text { Score† }\end{array}$ \\
\hline Grosset et al., 1992 & Pro & Clinical & 120 & 0 & 1 & 1 & 0 & 1 & 0 & 3 \\
\hline Vora et al., 1999 & Pro + retro & Clinical & 120 & 1 & 0 & 0 & 1 & 1 & 0 & 3 \\
\hline Rätsep \& Asser, 2001 & Pro & Clinical \pm CT & 120 & 0 & 1 & 1 & 1 & 1 & 1 & 5 \\
\hline Suarez et al., 2002 & Retro & Clinical & 120 & 1 & 1 & 1 & 1 & 1 & 0 & 5 \\
\hline Mascia et al., 2003 & Pro & $\begin{array}{c}\text { Clinical } \pm \\
\text { angio }\end{array}$ & 160 & 1 & 1 & 1 & 1 & 1 & 0 & 5 \\
\hline Rabinstein et al., 2004 & Retro & CT & 120 & 1 & 1 & 1 & 1 & 1 & 1 & 6 \\
\hline Smith et al., 2005 & Retro & Clinical & 120 & 1 & 1 & 1 & 1 & 1 & 0 & 5 \\
\hline Naval et al., 2005 & Retro & Clinical & 120 & 1 & 1 & 0 & 1 & 1 & 1 & 5 \\
\hline Sviri et al., 2006‡ & Retro & Clinical & 70 & 1 & 1 & 1 & 1 & 1 & 0 & 5 \\
\hline Lee et al., 2006 & Retro & CT & 120 & 1 & 1 & 1 & 1 & 1 & 0 & 5 \\
\hline Gonzalez et al., 2007 & Retro & Clinical & 120 & 1 & 1 & 1 & 1 & 1 & 0 & 5 \\
\hline Fontanella et al., 2008 & Pro & CT & 120 & 0 & 1 & 1 & 1 & 1 & 0 & 4 \\
\hline Carrera et al., $2009^{3}$ & Retro & Clinical or CT & 120 & 1 & 1 & 1 & 1 & 1 & 0 & 5 \\
\hline Nakae et al., 2011 & Retro & Clinical or CT & 125 & 1 & 1 & 1 & 1 & 1 & 1 & 6 \\
\hline Miller et al., 2011 & Retro & CT & 120 & 1 & 1 & 1 & 1 & 1 & 0 & 5 \\
\hline Sebastian et al., $2013 \S$ & Pro & Clinical & 120,150 & 1 & 1 & 1 & 1 & 1 & 0 & 5 \\
\hline Malhotra et al., 2014 & Retro & $\begin{array}{c}\text { Clinical + } \\
\text { angio }\end{array}$ & 120 & 1 & 1 & 1 & 1 & 1 & 0 & 5 \\
\hline
\end{tabular}

Angio = angiography; pro = prospective; retro = retrospective.

* For diagnosis of vasospasm on TCD.

$\dagger$ For each quality metric, the item received a score of 1 if the study fulfilled the criterion and 0 otherwise. The quality score was obtained by a sum of the individual scores in the row. The quality score is integrated into the regression to obtain quality-adjusted estimates (Table 2).

$\ddagger$ The only study on basilar artery vasospasm.

$\S$ Three criteria were used: 1) baseline middle cerebral artery (MCA) MFV $\geq 120 \mathrm{~cm} / \mathrm{sec}, 2)$ MCA MFV $\geq 150 \mathrm{~cm} / \mathrm{sec}$ before angiography, and 3) ratio of MCA MFV before angiography/baseline MCA MFV $\geq 1.5$.

erogeneity. Quantifiable heterogeneity did not disappear in the analysis of the subgroup that defined DCI clinically. Inclusion of radiography and angiography in the DCI definition by some of these studies is the likely explanation for persistent variance attributable to heterogeneity. Syntheses for all outcomes were robust to the exclusion sensitivity analysis, and exclusion of no single study influenced the significance of the pooled estimates.

\section{Discussion}

Our study is the first systematic review and meta-analysis in the literature that addresses the impact of TCD evidence of vasospasm on DCI, a clinically relevant out- come event in aSAH. We found that TCD evidence of vasospasm predicts DCI with high sensitivity, high NPV, and fair specificity. Our failure to find studies that address the impact of TCD monitoring on functional outcome and mortality underscores the need for patient-centered trials on comparative effectiveness of TCD. Our results indicate that vasospasm diagnosed on TCD can accurately predict DCI. High sensitivity and NPV make it ideal as a monitoring device, given low false negatives and a high probability that patients with a negative TCD truly have a low likelihood of developing DCI.

A large prospective study of TCD monitoring in aSAH came to this conclusion: ${ }^{52}$

TABLE 2. Pooled unadjusted and quality-adjusted estimates

\begin{tabular}{|c|c|c|c|c|c|c|c|c|c|}
\hline \multirow[b]{2}{*}{ Outcome } & \multirow[b]{2}{*}{$\mathrm{I}^{2}(\%)$} & \multirow[b]{2}{*}{$\tau^{2}$} & \multirow[b]{2}{*}{ MWE (95\% Cl) } & \multicolumn{4}{|c|}{ MWE $(95 \% \mathrm{Cl})$} & \multicolumn{2}{|l|}{$\tau^{2}$} \\
\hline & & & & Quality-Adjusted & Excluding BA Study & Radiological & Clinical & Radiological & Clinical \\
\hline Sensitivity & 99.3 & 0.89 & $90 \%(77-96)$ & $89 \%(88-90)$ & $91 \%(78-96)$ & $92 \%(58-99)$ & $89 \%(76-95)$ & 0.89 & 0.61 \\
\hline Specificity & 98.5 & 0.48 & $71 \%(51-84)$ & $74 \%(72-76)$ & $71 \%(50-84)$ & $71 \%(-1.5$ to 95$)$ & $71 \%(56-81)$ & 0.85 & 0.19 \\
\hline PPV & 97.4 & 0.24 & $57 \%(38-71)$ & $57 \%(54-60)$ & $57 \%(37-72)$ & $61 \%(19-85)$ & $54 \%(39-67)$ & 0.28 & 0.12 \\
\hline NPV* & 99.0 & 0.65 & $92 \%(83-96)$ & $91 \%(90-92)$ & $92 \%(83-96)$ & $93 \%$ (64-99) & $91 \%(83-96)$ & 0.81 & 0.38 \\
\hline
\end{tabular}

$\mathrm{BA}=$ basilar artery; $\mathrm{I}^{2}=$ percentage of total variance attributable to heterogeneity; $\tau^{2}=$ between-study variance.

* Publication bias was significant on Begg's test $(p=0.03)$ but not Egger's test. Trim and fill correction yielded publication bias-adjusted MWE of $94 \%$ ( $95 \%$ Cl $94 \%$ $95 \%)$. 

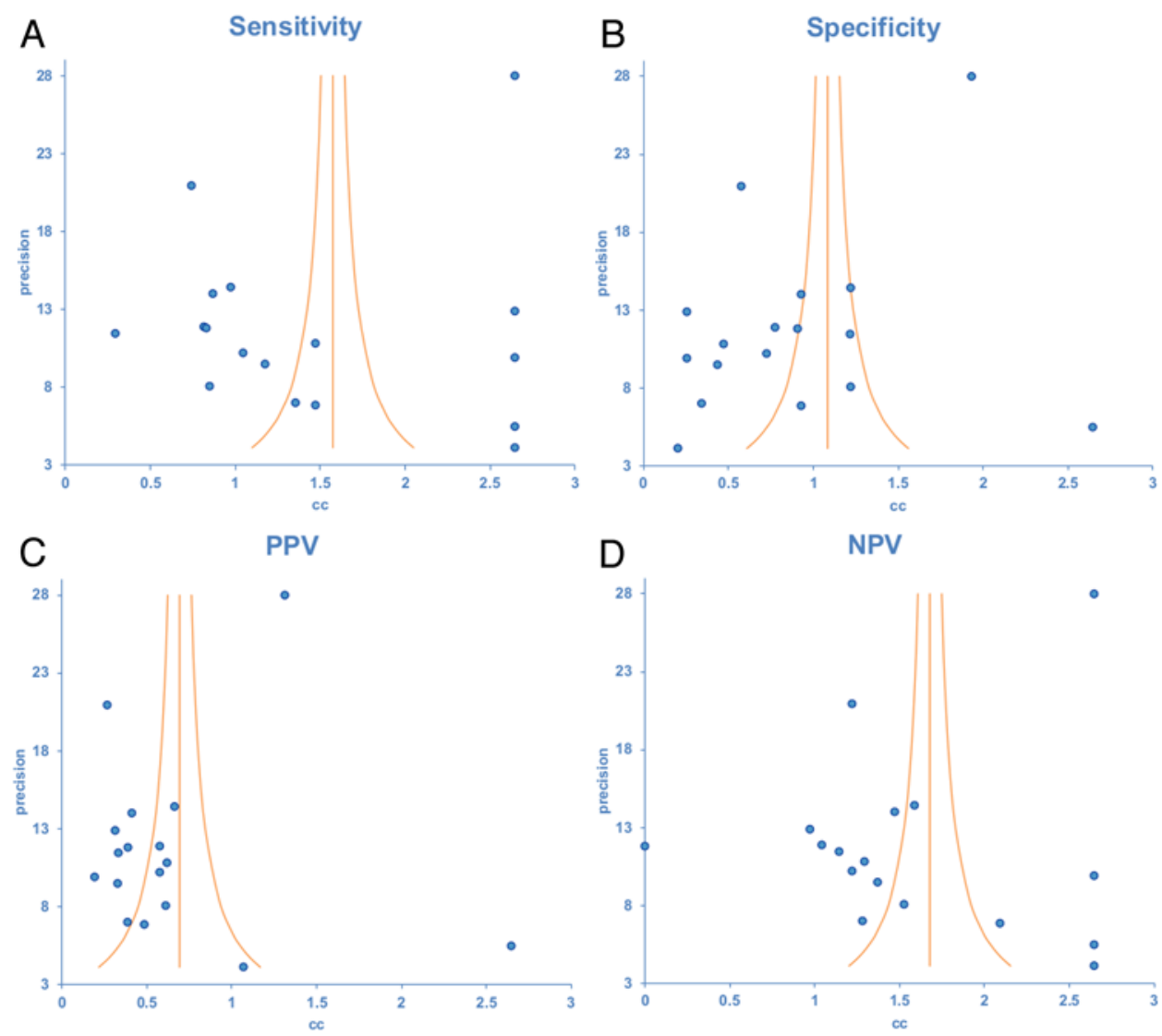

FIG. 2. Funnel plots for the syntheses of sensitivity (A), specificity (B), PPV (C), and NPV (D). Publication bias was not significant except for the synthesis of NPV ( $p=0.03$, Begg's test). Each study is represented by a single equally sized dot. With pseudo-Cls integrated into the plot (orange lines), it can be used to assess how heterogeneous the studies are and which studies are relative outliers. Inverse standard error is on the $y$-axis, and a measure of effect size (correlation coefficient [cc]) is on the x-axis. Figure is available in color online only.

A routine TCD service provided by neuroradiographers is accurate and useful in diagnosing and managing elevated blood velocities and ischemic neurological deficit following $\mathrm{SAH}$. In addition, it is possible that if the information gleaned from TCD findings was used more often in patient management, outcome might be improved; however, a randomized controlled trial is necessary to assess both these points definitively.

The study demonstrated that TCD was able to predict the subsequent development of symptomatic vasospasm and make a positive contribution to the diagnosis of vasospasm in $72 \%$ of patients. ${ }^{52}$ Our results suggest that poor clinical outcomes are potentially avoidable if vasospasm can be identified and treated before permanent cerebral injury sets in. Specific treatments to prevent and treat symptomatic vasospasm include nimodipine, hyperdynamic therapy with induced hypertension, augmentation of cardiac output using inotropic drugs, and intraarterial treatments such as luminal angioplasty and infusions of vasodilators such as nimodipine, verapamil, and milrinone. . $^{3,32,36,38,42}$ Despite knowledge that these treatments are effective against vasospasm, these are underutilized due to the absence of real-time monitoring that may detect vasospasm in the presymptomatic phase. With daily TCD monitoring these specific treatments could begin earlier to not only treat symptomatic vasospasm but also prevent va- sospasm from advancing to the symptomatic phase. Due to lack of direct evidence of impact on clinical outcomes, TCD monitoring is inconsistent and highly variable from institution to institution, and even within an institution among different providers. Therefore, equipoise still exists with regard to TCD monitoring in aSAH.

We posit that the evidence can be strengthened by conducting comparative effectiveness studies of TCD monitoring in aSAH. We believe the key is to design a pragmatic randomized study that tests relevant patient-centered and physician-centric outcomes with and without TCD monitoring. The focus of such work could be to establish that the use of TCD can shorten the time to diagnosis of subclinical and clinical vasospasm and improve outcomes relevant to patients and physicians such as timing of therapy, duration of intensive care unit stay, exposure to contrast agents/radiation, avoidance of ischemic injury, etc. The findings of our systematic review and meta-analysis highlight the need for future studies to evaluate the effectiveness of daily TCD monitoring on patient-centered outcomes in aSAH.

A previous systematic review/meta-analysis did not address the clinical impact of TCD evidence of vasospasm and was focused on correlation with angiographic vasospasm. ${ }^{24}$ Our study has various methodological strengths. 
A

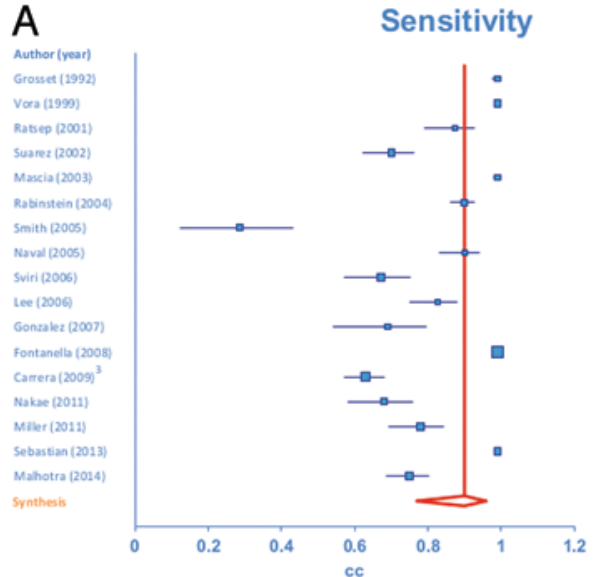

C

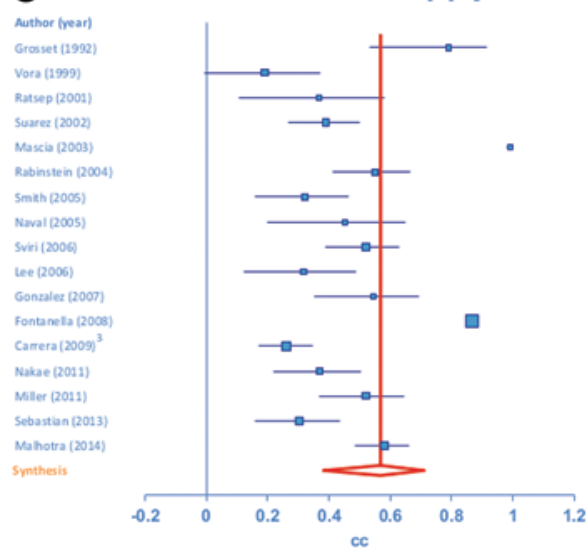

B
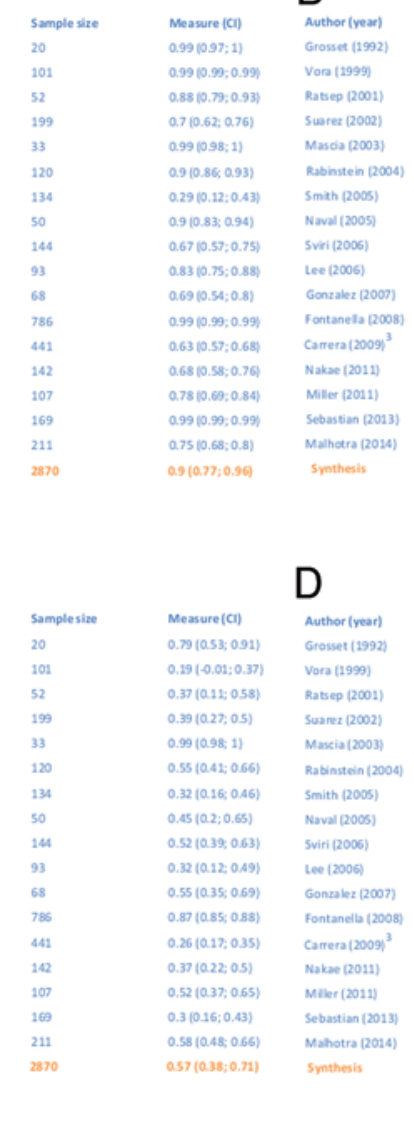

D

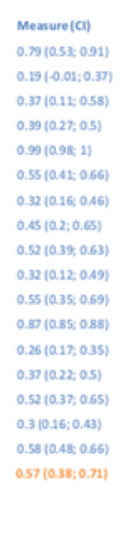

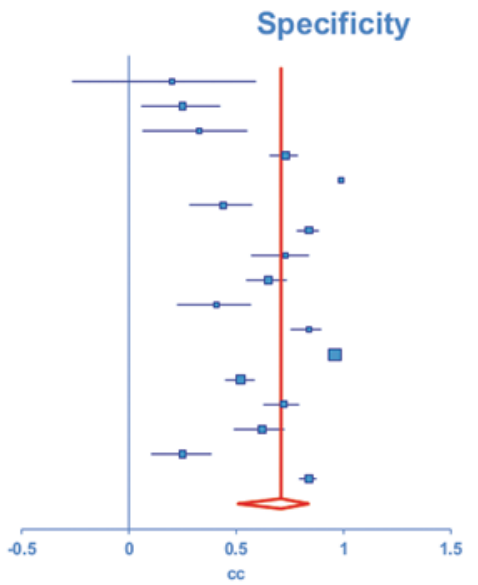

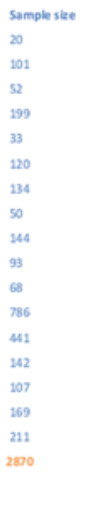

Measure (a) $0.2(0.23 ; 0.59)$ $0.25(0.06 ; 0.473$ $0.73(0.66 ; 0.79)$ $0.73(0.66 ; 0.79 \%$ $0.4410 .28 ; 0.57$ $0.8410 .78 ; 0.88$ $0.7310 .57: 0.84$ 0.65 (10.54: 0.74) $0.410 .23 ; 0.57$ $0.84(0.75 ; 0.9)$ $0.9610 .95 ; 0.96$ 0.52 (10.45: 0.58; $0.20090 ; 072$ $0.25(0.939$

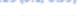
$0.71(0.51 ; 0.98)$

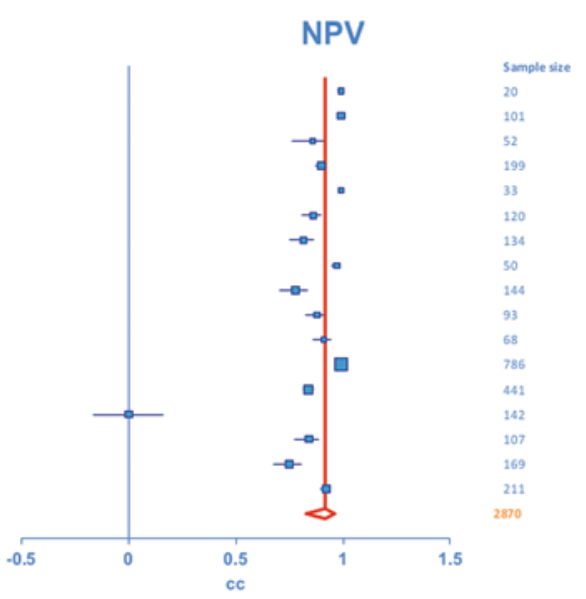

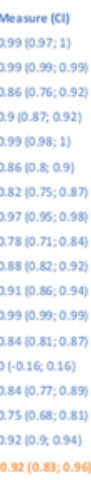

FIG. 3. Forest plots for the syntheses of sensitivity (A), specificity (B), PPV (C), and NPV (D). cc = correlation coefficient. Figure is available in color online only.

Unlike the previous meta-analysis, our study employed appropriate statistical methods to address the issues of publication bias, study quality, and heterogeneity. We performed a priori-designed subgroup analyses and identified that the way DCI was defined (i.e., clinical vs radiological) explained heterogeneity. Heterogeneity was lower in the subgroup that used a clinical definition of DCI and greater in the subgroup that used radiological/angiographic means to diagnose DCI. This finding uncovers the variation in the definitions used for diagnosing DCI in the literature and highlights the need for future studies to consistently follow a consensus definition of DCI.$^{50}$ To adjust for heterogeneity from variation in quality across studies, we performed quality adjustment that did not sizably change the pooled estimates of sensitivity, specificity, PPV, and NPV. We evaluated and accounted for publication bias, a major threat to the validity of observational study meta-analyses. Publication bias was not significant except in the synthesis of NPV, for which trim and fill correction led to tighter CIs. We demonstrated robustness of our pooled estimates with exclusion sensitivity analysis.

Our study has several limitations. Observational studies lack the inherent bias elimination fail-safe of randomization. Meta-analyses of randomized trials are based on the assumption that each trial provides an unbiased estimate of treatment effect, with the variability between study re- sults attributable to random variation. Conversely, observational studies yield estimates that may deviate from true underlying relationships beyond the incidence of chance. A meta-analysis of such studies has the potential for bias. However, thorough consideration of possible sources of heterogeneity between study results provides more insights than the mechanistic calculation of an overall measure of effect, which could be biased.

Although we attempted to adhere closely to the consensus definition of DCI, ${ }^{50}$ because most studies included in the analysis were published prior to the publication of the consensus guideline, studies that used definitions that did not strictly conform to the recommendation were also included (Table 1). This serves to account for heterogeneity. We had to exclude the Lindegaard ratio as a diagnostic criterion for vasospasm because most studies did not use it. Instead, to ensure comparability we used the MFV of $120 \mathrm{~cm} / \mathrm{sec}$ as the cutoff for diagnosis used by most studies. This is a drawback of our study because distinction between vasospasm and hyperemia is reliably made only with calculation of the Lindegaard ratio. We did not seek angiographic confirmation of vasospasm and used TCD evidence of vasospasm..$^{48}$ The reason for this was 2-fold. First, we sought to evaluate the clinical impact of vasospasm as diagnosed by TCD. The dynamic monitoring capability allowing daily surveillance of asymptomatic 
patients (those in subclinical vasospasm), coupled with the physiological information generated that confers on TCD the ability to predict symptomatic vasospasm, make it a unique modality that does not justify nor allow a gold-standard confirmation. Second, since there is still variation in the TCD definition of vasospasm, we used the MFV cutoff that is most widely accepted to allow an apples-to-apples comparison.

Sources of heterogeneity other than the way DCI and vasospasm are defined exist. Variation in the treatment of vasospasm introduces heterogeneity. No guidelines currently exist for the treatment of vasospasm. Induced hypertension and hyperdynamic therapy are variably employed for treatment. Nimodipine and statin use can influence outcomes. The year of publication is a potential source of heterogeneity since health care and technological improvements over time may result in improved outcomes. Country of origin is a source of heterogeneity as practice patterns, guidelines, and protocols vary between countries. Quality metrics other than what this study accounted for are potential sources of heterogeneity. These metrics include variation in the time of outcome assessment, variation in the definition of vasospasm, sonographer skill and experience, severity of subarachnoid hemorrhage, etc. These factors need to be carefully considered when designing a trial to study the impact on outcomes.

\section{Conclusions}

TCD evidence of vasospasm is predictive of DCI with high accuracy. Although high sensitivity and NPV make TCD an ideal monitoring device, it is not a mandated standard of care in aSAH due to the paucity of evidence on clinically relevant outcomes despite recommendation by national guidelines. High-quality randomized trials evaluating the impact of TCD monitoring on patient-centered and physician-relevant outcomes are needed.

\section{References}

1. Aaslid R, Huber P, Nornes H: Evaluation of cerebrovascular spasm with transcranial Doppler ultrasound. J Neurosurg 60:37-41, 1984

2. Begg CB, Mazumdar M: Operating characteristics of a rank correlation test for publication bias. Biometrics 50:10881101, 1994

3. Carrera E, Schmidt JM, Oddo M, Fernandez L, Claassen J, Seder D, et al: Transcranial Doppler for predicting delayed cerebral ischemia after subarachnoid hemorrhage. Neurosurgery 65:316-324, 2009

4. Carrera E, Schmidt JM, Oddo M, Ostapkovich N, Claassen J, Rincon F, et al: Transcranial Doppler ultrasound in the acute phase of aneurysmal subarachnoid hemorrhage. Cerebrovasc Dis 27:579-584, 2009

5. Connolly ES Jr, Rabinstein AA, Carhuapoma JR, Derdeyn CP, Dion J, Higashida RT, et al: Guidelines for the management of aneurysmal subarachnoid hemorrhage: a guideline for healthcare professionals from the American Heart Association/American Stroke Association. Stroke 43:1711-1737, 2012

6. DerSimonian R, Laird N: Meta-analysis in clinical trials. Control Clin Trials 7:177-188, 1986

7. Diringer MN, Bleck TP, Hemphill JC III, Menon D, Shutter L, Vespa P, et al : Critical care management of patients following aneurysmal subarachnoid hemorrhage: recommenda- tions from the Neurocritical Care Society's Multidisciplinary Consensus Conference. Neurocrit Care 15:211-240, 2011

8. Doi SA, Thalib L: A quality-effects model for meta-analysis. Epidemiology 19:94-100, 2008

9. Duval S, Tweedie R: Trim and fill: A simple funnel-plotbased method of testing and adjusting for publication bias in meta-analysis. Biometrics 56:455-463, 2000

10. Egger M, Davey Smith G, Schneider M, Minder C: Bias in meta-analysis detected by a simple, graphical test. BMJ 315: 629-634, 1997

11. Fergusen S, Macdonald RL: Predictors of cerebral infarction in patients with aneurysmal subarachnoid hemorrhage. Neurosurgery 60:658-667, 2007

12. Fisher CM, Roberson GH, Ojemann RG: Cerebral vasospasm with ruptured saccular aneurysm-the clinical manifestations. Neurosurgery 1:245-248, 1977

13. Fontanella M, Valfrè W, Benech F, Carlino C, Garbossa D, Ferrio M, et al: Vasospasm after SAH due to aneurysm rupture of the anterior circle of Willis: value of TCD monitoring. Neurol Res 30:256-261, 2008

14. Fraticelli AT, Cholley BP, Losser MR, Saint Maurice JP, Payen D: Milrinone for the treatment of cerebral vasospasm after aneurysmal subarachnoid hemorrhage. Stroke 39:893-898, 2008

15. Gonzalez NR, Boscardin WJ, Glenn T, Vinuela F, Martin NA: Vasospasm probability index: a combination of transcranial Doppler velocities, cerebral blood flow, and clinical risk factors to predict cerebral vasospasm after aneurysmal subarachnoid hemorrhage. J Neurosurg 107:1101-1112, 2007

16. Grosset DG, Straiton J, du Trevou M, Bullock R: Prediction of symptomatic vasospasm after subarachnoid hemorrhage by rapidly increasing transcranial Doppler velocity and cerebral blood flow changes. Stroke 23:674-679, 1992

17. Harris RP, Helfand M, Woolf SH, Lohr KN, Mulrow CD, Teutsch SM, et al : Current methods of the US Preventive Services Task Force: a review of the process. Am J Prev Med 20 (3 Suppl):21-35, 2001

18. Huedo-Medina TB, Sánchez-Meca J, Marín-Martínez F, Botella J: Assessing heterogeneity in meta-analysis: Q statistic or I2 index? Psychol Methods 11:193-206, 2006

19. Kassell NF, Haley EC Jr, Apperson-Hansen C, Alves WM: Randomized, double-blind, vehicle-controlled trial of tirilazad mesylate in patients with aneurysmal subarachnoid hemorrhage: a cooperative study in Europe, Australia, and New Zealand. J Neurosurg 84:221-228, 1996

20. Kassell NF, Sasaki T, Colohan AR, Nazar G: Cerebral vasospasm following aneurysmal subarachnoid hemorrhage. Stroke 16:562-572, 1985

21. Keyrouz SG, Diringer MN: Clinical review: Prevention and therapy of vasospasm in subarachnoid hemorrhage. Crit Care 11:220, 2007

22. Lee JY, Lee MS, Whang K, Lee JM, Kim SH, Lee SS: Accuracy of transcranial Doppler sonography for predicting cerebral infarction in aneurysmal subarachnoid hemorrhage. J Clin Ultrasound 34:380-384, 2006

23. Lindegaard KF: The role of transcranial Doppler in the management of patients with subarachnoid haemorrhage-a review. Acta Neurochir Suppl 72:59-71, 1999

24. Lysakowski C, Walder B, Costanza MC, Tramèr MR: Transcranial Doppler versus angiography in patients with vasospasm due to a ruptured cerebral aneurysm: a systematic review. Stroke 32:2292-2298, 2001

25. Macdonald RL, Hunsche E, Schüler R, Wlodarczyk J, Mayer SA: Quality of life and healthcare resource use associated with angiographic vasospasm after aneurysmal subarachnoid hemorrhage. Stroke 43:1082-1088, 2012

26. Macdonald RL, Kassell NF, Mayer S, Ruefenacht D, Schmiedek P, Weidauer S, et al: Clazosentan to overcome neurological ischemia and infarction occurring after subarachnoid hemorrhage (CONSCIOUS-1): randomized, double-blind, 
placebo-controlled phase 2 dose-finding trial. Stroke 39:3015-3021, 2008

27. Malhotra K, Conners JJ, Lee VH, Prabhakaran S: Relative changes in transcranial Doppler velocities are inferior to absolute thresholds in prediction of symptomatic vasospasm after subarachnoid hemorrhage. J Stroke Cerebrovasc Dis 23:31-36, 2014

28. Manno EM, Gress DR, Schwamm LH, Diringer MN, Ogilvy CS: Effects of induced hypertension on transcranial Doppler ultrasound velocities in patients after subarachnoid hemorrhage. Stroke 29:422-428, 1998

29. Mascia L, Fedorko L, terBrugge K, Filippini C, Pizzio M, Ranieri VM, et al: The accuracy of transcranial Doppler to detect vasospasm in patients with aneurysmal subarachnoid hemorrhage. Intensive Care Med 29:1088-1094, 2003

30. Miller CM, Palestrant D, Schievink WI, Alexander MJ: Prolonged transcranial Doppler monitoring after aneurysmal subarachnoid hemorrhage fails to adequately predict ischemic risk. Neurocrit Care 15:387-392, 2011

31. Moppett IK, Mahajan RP: Transcranial Doppler ultrasonography in anaesthesia and intensive care. Br J Anaesth 93:710-724, 2004

32. Muizelaar JP, Becker DP: Induced hypertension for the treatment of cerebral ischemia after subarachnoid hemorrhage. Direct effect on cerebral blood flow. Surg Neurol 25:317325,1986

33. Nakae R, Yokota H, Yoshida D, Teramoto A: Transcranial Doppler ultrasonography for diagnosis of cerebral vasospasm after aneurysmal subarachnoid hemorrhage: mean blood flow velocity ratio of the ipsilateral and contralateral middle cerebral arteries. Neurosurgery 69:876-883, 2011

34. Naval NS, Thomas CE, Urrutia VC: Relative changes in flow velocities in vasospasm after subarachnoid hemorrhage: a transcranial Doppler study. Neurocrit Care 2:133-140, 2005

35. Neil-Dwyer G, Mee E, Dorrance D, Lowe D: Early intervention with nimodipine in subarachnoid haemorrhage. Eur Heart J 8 (Suppl K):41-47, 1987

36. Otsubo H, Takemae T, Inoue T, Kobayashi S, Sugita K: Normovolaemic induced hypertension therapy for cerebral vasospasm after subarachnoid haemorrhage. Acta Neurochir (Wien) 103:18-26, 1990

37. Peters JL, Sutton AJ, Jones DR, Abrams KR, Rushton L: Comparison of two methods to detect publication bias in meta-analysis. JAMA 295:676-680, 2006

38. Polin RS, Coenen VA, Hansen CA, Shin P, Baskaya MK, Nanda A, et al: Efficacy of transluminal angioplasty for the management of symptomatic cerebral vasospasm following aneurysmal subarachnoid hemorrhage. J Neurosurg 92:284290, 2000

39. Rabinstein AA, Friedman JA, Weigand SD, McClelland RL, Fulgham JR, Manno EM, et al: Predictors of cerebral infarction in aneurysmal subarachnoid hemorrhage. Stroke 35:1862-1866, 2004

40. Rätsep T, Asser T: Cerebral hemodynamic impairment after aneurysmal subarachnoid hemorrhage as evaluated using transcranial Doppler ultrasonography: relationship to delayed cerebral ischemia and clinical outcome. J Neurosurg 95:393-401, 2001

41. Sebastian J, Derksen C, Khan K, Ibrahim M, Hameed B, Siddiqui M, et al: Derivation of transcranial Doppler criteria for angiographically proven middle cerebral artery vasospasm after aneurysmal subarachnoid hemorrhage. J Neuroimaging 23:489-494, 2013

42. Sehy JV, Holloway WE, Lin SP, Cross DT III, Derdeyn CP, Moran CJ: Improvement in angiographic cerebral vasospasm after intra-arterial verapamil administration. AJNR Am J Neuroradiol 31:1923-1928, 2010
43. Sloan MA, Alexandrov AV, Tegeler CH, Spencer MP, Caplan LR, Feldmann E, et al: Assessment: transcranial Doppler ultrasonography: report of the Therapeutics and Technology Assessment Subcommittee of the American Academy of Neurology. Neurology 62:1468-1481, 2004

44. Smith ML, Abrahams JM, Chandela S, Smith MJ, Hurst RW, Le Roux PD: Subarachnoid hemorrhage on computed tomography scanning and the development of cerebral vasospasm: the Fisher grade revisited. Surg Neurol 63:229-235, 2005

45. Stroup DF, Berlin JA, Morton SC, Olkin I, Williamson GD, Rennie D, et al: Meta-analysis of observational studies in epidemiology: a proposal for reporting. Meta-analysis Of Observational Studies in Epidemiology (MOOSE) group. JAMA 283:2008-2012, 2000

46. Suarez JI, Qureshi AI, Yahia AB, Parekh PD, Tamargo RJ, Williams MA, et al: Symptomatic vasospasm diagnosis after subarachnoid hemorrhage: evaluation of transcranial Doppler ultrasound and cerebral angiography as related to compromised vascular distribution. Crit Care Med 30:1348-1355, 2002

47. Sviri GE, Ghodke B, Britz GW, Douville CM, Haynor DR, Mesiwala AH, et al: Transcranial Doppler grading criteria for basilar artery vasospasm. Neurosurgery 59:360-366, 2006

48. Vergouwen MD: Vasospasm versus delayed cerebral ischemia as an outcome event in clinical trials and observational studies. Neurocrit Care 15:308-311, 2011

49. Vergouwen MD, Ilodigwe D, Macdonald RL: Cerebral infarction after subarachnoid hemorrhage contributes to poor outcome by vasospasm-dependent and -independent effects. Stroke 42:924-929, 2011

50. Vergouwen MD, Vermeulen M, van Gijn J, Rinkel GJ, Wijdicks EF, Muizelaar JP, et al: Definition of delayed cerebral ischemia after aneurysmal subarachnoid hemorrhage as an outcome event in clinical trials and observational studies: proposal of a multidisciplinary research group. Stroke 41:2391-2395, 2010

51. Vora YY, Suarez-Almazor M, Steinke DE, Martin ML, Findlay JM: Role of transcranial Doppler monitoring in the diagnosis of cerebral vasospasm after subarachnoid hemorrhage. Neurosurgery 44:1237-1248, 1999

52. Wardlaw JM, Offin R, Teasdale GM, Teasdale EM: Is routine transcranial Doppler ultrasound monitoring useful in the management of subarachnoid hemorrhage? J Neurosurg 88:272-276, 1998

\section{Disclosure}

The authors report no conflict of interest concerning the materials or methods used in this study or the findings specified in this paper.

\section{Author Contributions}

Conception and design: Kumar. Acquisition of data: Kumar, Bavarsad Shahripour. Analysis and interpretation of data: Kumar. Drafting the article: Kumar. Critically revising the article: all authors. Reviewed submitted version of manuscript: all authors. Approved the final version of the manuscript on behalf of all authors: Kumar. Statistical analysis: Kumar. Administrative/ technical/material support: Kumar. Study supervision: Kumar, Harrigan.

\section{Correspondence}

Gyanendra Kumar, Stroke Center, University of Alabama Hospital, 1813 6th Ave. South, RWUH M226, Birmingham, AL 35249. email: kumarg@uab.edu. 